\title{
Wideband Absorption Enhancement in Laterally Oriented Core-Shell c-Si/a-Si Hexagonal Nanowire Arrays
}

\author{
Mojtaba Shahraki* and Majid Ghadrdan \\ Faculty of Electrical and Computer Engineering, University of Sistan and Baluchestan, \\ Zahedan, IRAN
}

*Corresponding author email: m.shahraki@ece.usb.ac.ir

Regular paper: Received: Jul. 12, 2020, Revised: Mar. 8, 2021, Accepted: May. 21, 2021, Available Online: May. 23, 2021, DOI: 10.52547/ijop.15.1.19

\begin{abstract}
In this paper, the optical properties of laterally oriented core-shell nanowire silicon solar cells (NWSCs) are optimized. The optimum structure consists of an array with non-uniform hexagonal nanowires (NWs). Each NW is constructed from an amorphous silicon layer sandwiched between two crystalline silicon layers. In order to improve the light absorption and short circuit current density $\left(J_{s c}\right)$ of NWSC, a particle swarm optimization (PSO) algorithm is used to optimize the geometrical parameters of NWs. It is shown that the optimized structure has advantageous performance in terms of light absorption and $J_{s c}$. Finally, a multiple structure composed of two NWs with different morphologies and the optimized dimensions is proposed to utilize NWSCs better.
\end{abstract}

KEYWORDS: core-shell Nanowires; amorphous silicon; crystalline silicon; whispering-gallery; thin films.

\section{I.INTRODUCTION}

In recent years, semiconducting nanowires (NWs) have attracted much attention in optical waveguides [1], low threshold lasers [2], and solar cells (SCs) [3]. They have shown unique optical properties, including large scattering cross-section [4], [5], low reflection [6], high absorption [7], spectral [8], [9], and polarization selectivity [10]. NW is also a lowcost structure to design SCs; however, the main challenge is to enhance the absorption and short circuit current density $\left(J_{s c}\right)$ of nanowire solar cells (NWSCs). In fact, the short circuit current density of these structures is relatively low due to the reduction of raw material. Crystalline silicon (c-Si) is the prominent material to design SCs, and around 90 percent of SCs on the market are based on c-Si [11]. The main drawback of $\mathrm{c}-\mathrm{Si}$ is the low absorption coefficient. On the contrary, amorphous silicon (a-Si) has a higher absorption coefficient, and it is considered an effective material to increase the NWSCs absorption [12]. One of the amorphous silicon imperfections is low diffusion length $(\sim 100 \mathrm{~nm})$ [7], which degrade the electrical properties of a-Si NWSCs. In order to overcome this limitation, the amorphous /crystalline silicon core/shell structures are simultaneously used to take advantage of the a-Si high absorption and the high diffusion length $(>200 \mu \mathrm{m})$ of c-Si [7]. Recently, vertically oriented NWSCs have been widely investigated, either as single NWs [13], [14] or NW arrays [15]-[21]. Nevertheless, in all studies based on the laterally oriented NWSCs, most of the attention was focused on the single NWSCs [22]-[24]. The number and the type of absorption resonance modes are the same for the single NW and NW arrays [24][27], while the position and amplitude resonance modes are different [25]. In this paper, simulations are based on the NW arrays, considering the fact that the real NWSCs are more in compliance with the NW arrays [14], [33]-[34].

One way to improve the absorption profile inside NWs is designing unusual crosssectional geometries. In recent years, so many 
efforts have been made to achieve broadband absorption enhancement in NWSCs by this method including, crescent-deformed [28], Nanos-spring [29] irregular [30], gear-shaped [31], and funnel-shaped NWs [32]. These nanostructures can achieve unique modes that are not supported in uniform and regular NWs. As a result, the absorption profile and the $J_{s c}$ of the SC can be improved considerably.

In the framework of the unusual structures, it is shown that non-uniform core-shell c-Si/a-Si hexagonal NWs can accomplish broadband absorption with the proper design of different layers. It is found that changing the crosssectional geometry of NWs from a uniform shape to non-uniform can adjust the absorption spectra and $J_{s c}$ of the NWSC. Another method to improve the absorption of the NWSCs is by introducing multiple NWs. This method has also been utilized extensively to enhance the $J_{s c}$ of NWSCs [16]-[18], [35]. In this case, it is possible to employ the resonances of different NWs, which results in the wideband absorption inside the SC.

In this paper, we have utilized this method to increase the $J_{S C}$ of core-shell c-Si/a-Si hexagonal NWs. It has been found that using two non-uniform NWs with different crosssectional geometries results in the absorption enhancement of NWSCs.

This study aims to determine the optimal structure for nanowires by studying the changes in the geometry and arrangement of nanowires on solar cells' performance. The considered nanowires are hexagonal, and the core-shell c$\mathrm{Si} / \mathrm{a}-\mathrm{Si}$ structure for these NWs is proposed. Using this structure, it is possible to simultaneously use the advantage of a higher absorption rate in a-Si layer and high diffusion length of c-Si layers. However, to prevent the detrimental effects of the a-Si layer on the electrical properties of the SCs, the thickness of this layer is limited to $20 \mathrm{~nm} \mathrm{[14].} \mathrm{The}$ geometry and arrangement of hexagonal NWs are changed and the effects of these changes on the absorption profile of SCs are studied. Several structures with various degrees of freedom for different parameters are proposed.
All of these structures are optimized using the PSO algorithm. As a final point, multiple structures consisting of two different NWs with different cross-sections and different arrangements are proposed and optimized, which led to considerable absorption of SCs.

The remainder of this paper is organized as follows. The theoretical model is described in Section 2. The non-uniform and multiple NWs are studied and optimized in Sections 3 and 4, respectively. Finally, a brief conclusion is provided in Section 5.

\section{TheORETICAL ModeL}

The uniform three-layer hexagonal NW structure is shown in Fig. 1(a), where the middle layer of a-Si is utilized to enhance the absorption inside the NWSC. Because of the low diffusion length of a-Si, this layer's thickness is fixed to $20 \mathrm{~nm}$ [14]. In this case, the detrimental effects of a-Si on the electrical properties of NWSC are negligible and can be ignored.

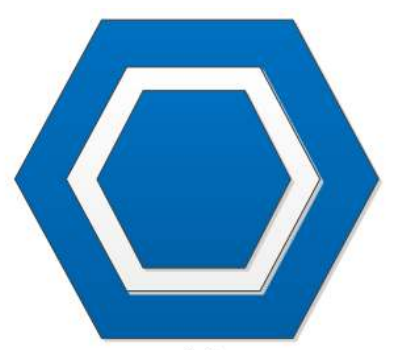

(a)

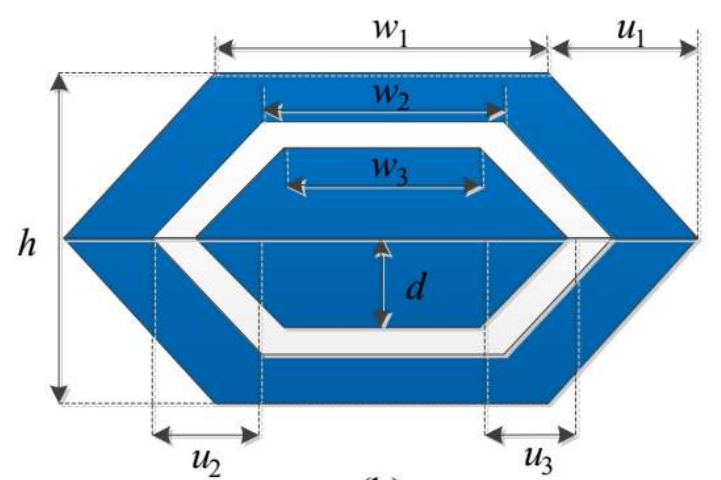

(b)

Fig. 1. Cross-sectional view of (a) uniform, (b) nonuniform NW.

Since the position and the amplitude of the resonance modes in the NWs absorption spectra are highly dependent on the NW geometry and 
dimension [24], [34], a non-uniform hexagonal structure is proposed, in which some changes are made in the NW cross-section. The effects of these changes on the absorption spectra and the $J_{s c}$ of the NWSC are investigated, and an optimization procedure is conducted to optimize the $J_{s c}$ of NWSC. To compare the proposed scheme with thin-film SCs, the total thicknesses of all NWs are assumed to be 200 $\mathrm{nm}$. In addition, the thickness of the a-Si layer is set to $20 \mathrm{~nm}$ to mitigate the negative impacts of a-Si on the electrical properties of NWSC [14]. In order to simulate a practical case of the $\mathrm{SC}$, arrays of the above-mentioned hexagonal NWs are placed on top of a sufficiently thick layer of quartz [25], [30].

To compare the proposed scheme with thin-film $\mathrm{SCs}$, the total thicknesses of all NWs are assumed to be $200 \mathrm{~nm}$. In addition, the thickness of the a-Si layer is set to $20 \mathrm{~nm}$ to mitigate the negative impacts of a-Si on the electrical properties of NWSC [14]. In order to simulate a practical case of the $\mathrm{SC}$, arrays of the above-mentioned hexagonal NWs are placed on top of a sufficiently thick layer of quartz [25], [30].

It is assumed that the sunlight is evenly composed of the Transverse Electric (TE) and the Transverse Magnetic (TM) polarizations. Moreover, the sunlight is emitted constantly on the NWSC surface. The refractive index of c$\mathrm{Si}$ and a-Si are taken from [12], and the simulations are based on the finite element method (FEM). In order to evaluate the proposed structure, the absorption spectrum is calculated for both TE and TM polarizations in uniform and non-uniform NWs. Then, assuming the unity of the internal quantum efficiency [19], the $J_{s c}$ is calculated based on the following equation [18]:

$J_{S c}=\int_{\operatorname{lam} 1}^{\operatorname{lam} 2} \frac{q \lambda}{h c} I(\lambda) A(\lambda) d \lambda$,

where $q$ is the electron charge, $\lambda$ is the radiation photon wavelength, $h$ is the Plank constant, and $c$ is the light velocity. $\operatorname{Lam}_{1}$ and $\operatorname{lam}_{2}$ are the minimum $(280 \mathrm{~nm})$ and the maximum wavelengths $(1000 \mathrm{~nm})$ respectively. $I(\lambda)$ is the standard radiation intensity of the sunlight spectrum on the earth, equal to $A M 1.5$, and $A(\lambda)$ is the SCs absorption.

\section{UNIFORM/NON-UNIFORM NWSCS}

The optical properties of c-Si and a_Si are taken from [12], and the correlation between bandgap and optical properties for different layers of NWSCs are shown in Fig. 2.

By applying Equation 1, the $J_{s c}$ curve of the uniform hexagonal NW is plotted in Fig. 3 as a function of the a-Si layer distance from the center to the shell $(d)$.
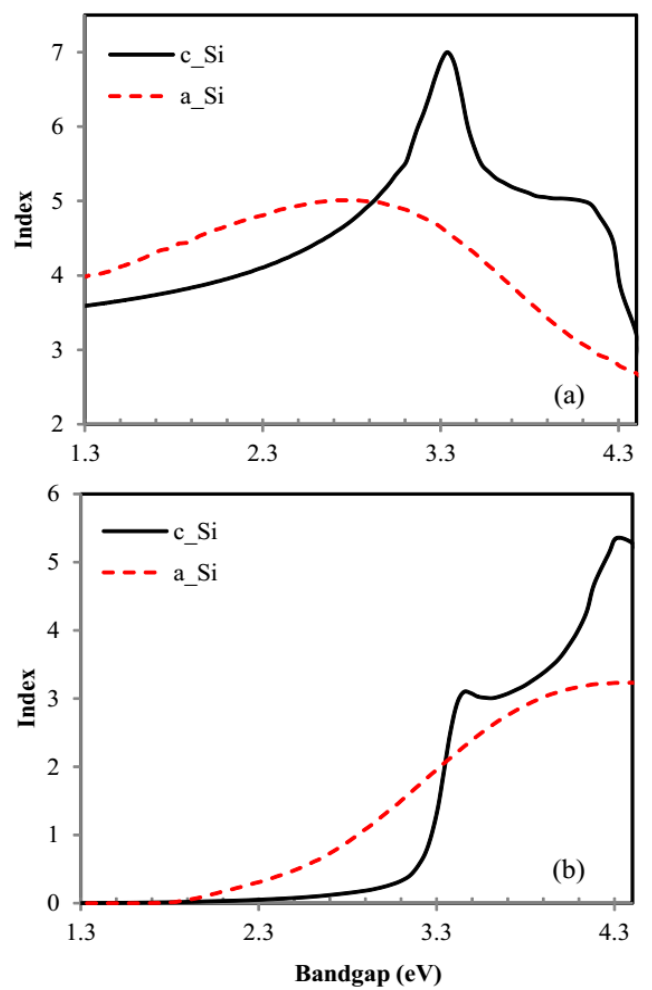

Fig. 2. The correlation between bandgap and optical properties for different layers of NWSCs, a) real part and $b$ ) imaginary part of refractive indices.

Based on this figure, the maximum value of the $J_{s c}$ is computed as $8.43 \mathrm{~mA} / \mathrm{cm}^{2}$, for $d=35 \mathrm{~nm}$. However, when the a-Si layer is in the center of the structure $(d=10 \mathrm{~nm})$, the minimum value of the $J_{s c}$ is obtained $\left(7.68 \mathrm{~mA} / \mathrm{cm}^{2}\right)$. There is a $9.76 \%$ difference between maximum and minimum of $J_{s c}$, based on the a-Si layer position changes. Therefore, optimization is essential.

In order to improve the light absorption and $J_{s c}$ of NWSC, a particle swarm optimization (PSO) 
algorithm is applied to find the optimized geometrical parameters of non-uniform NWs. In this case, the variables are chosen to be $d, w_{1}$, $w_{2}$, and $w_{3}$ (Fig. 1 (b)).

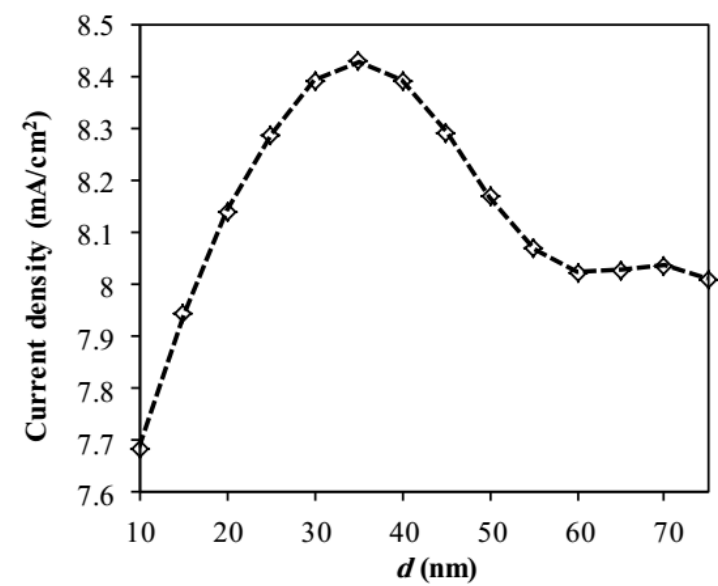

Fig. 3. $J_{s c}$ of a uniform NWSC with a total thickness of $200 \mathrm{~nm}$ as a function of a-Si layer distance from the center.

The absorption spectra of the optimized nonuniform structure are shown in Fig.4 for both $\mathrm{TE}$ and TM polarizations and compared to the uniform NWSC and the thin film SC with the same thickness of c-Si and a-Si layers. To have a better comparison of the proposed structure with thin film SCs, this-film has the same thickness of a-Si, while the position of the a-Si layer is optimized as well. The non-uniform NW arrays have the higher absorption for the most wavelengths. However, at some parts of the wavelength spectra (lower than $400 \mathrm{~nm}$ ) in the TM polarization, the absorption intensity of uniform NW is higher than non-uniform NW.

In order to have a more detailed discussion on this feature, the sunlight intensity (AM 1.5) alongside the absorption spectra of the uniform and non-uniform arrays for TM polarization is displayed in Fig. 5. The radiation intensity of the sunlight is dropped abruptly for the lower wavelengths. So, the absorption intensity for these wavelengths does not significantly influence the $J_{S C}$ of the SC.

Referring to Fig. 5, broadband absorption is obtained, and a new resonance mode is generated in the wavelength of $919 \mathrm{~nm}$ for TE polarization in the proposed non-uniform structure. Since in this wavelength domain, the uniform NW's absorption intensity is almost zero, generating the new resonance mode in the non-uniform structure definitely shows the effect of the optimized design. There is a $19 \%$ increase in the $J_{S C}$ compared to the optimized uniform Core-Shell c-Si/a-Si NWs.

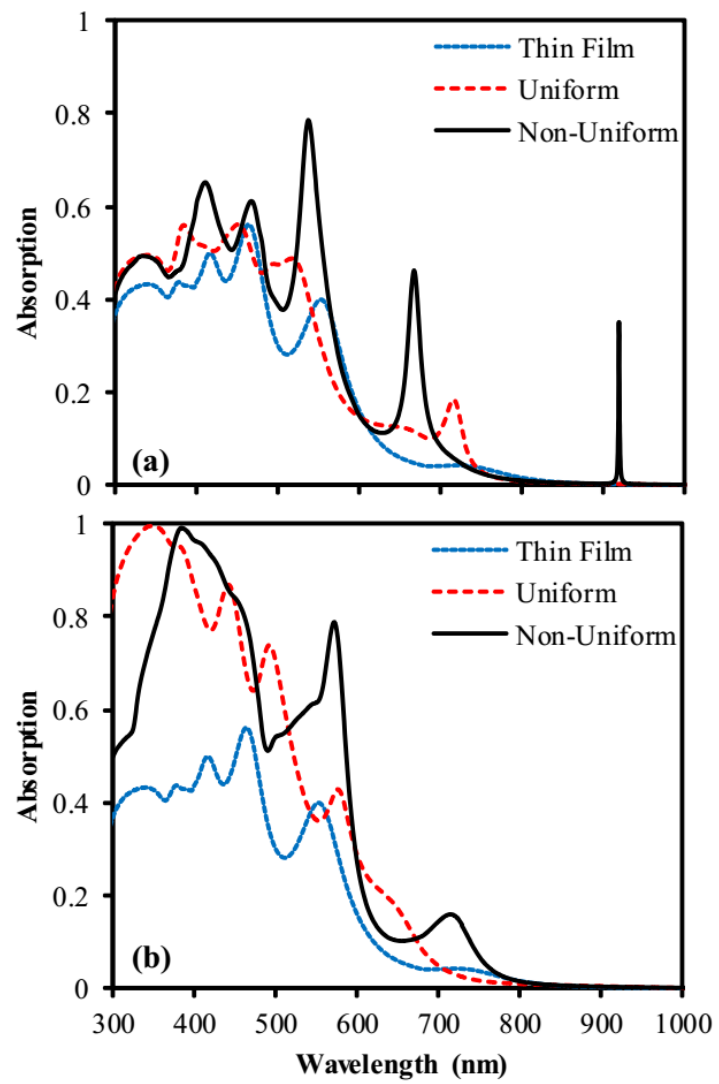

Fig. 4. Absorption spectra of uniform and nonuniform NWs as well as thin-film SCs for (a) TE and (b) TM polarization.

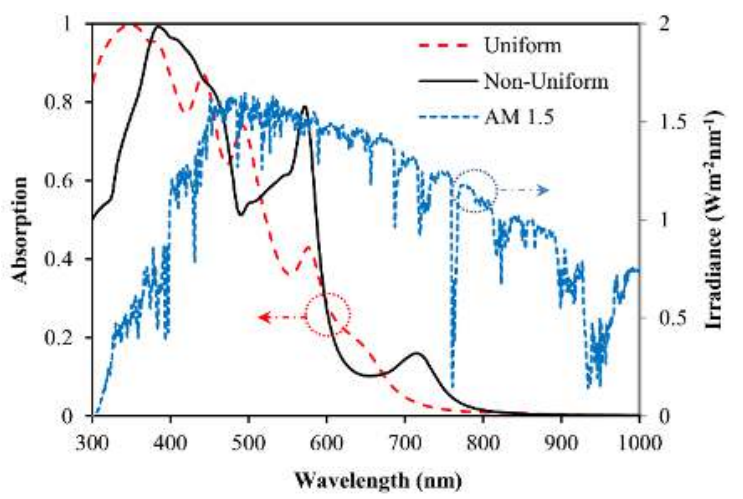

Fig. 5. Overlap figure of the absorption intensity and the radiation intensity of the sun spectrum (AM1.5).

Also, for the non-uniform C-Si NWs and crescent-deformed solar cells, the optimized $J_{S C}$ is 7.32 [30] and $7.94 \mathrm{~mA} / \mathrm{cm} 2$ [35], which are 
$27 \%$ and $17 \%$ lower than our proposed structure, respectively.

To determine the type of the resonance modes, the electric field intensity profile in the TE absorption spectrum peaks and the magnetic field intensity of the TM absorption spectrum peaks are shown in Fig. 6.

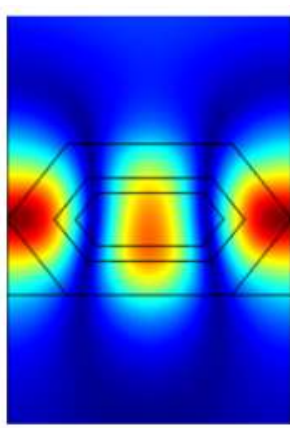

(a)

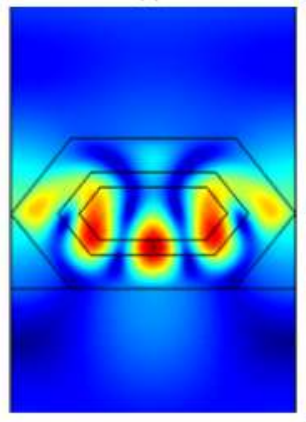

(c)

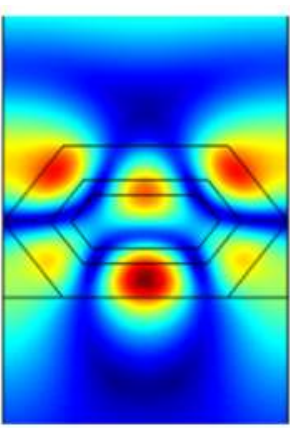

(b)

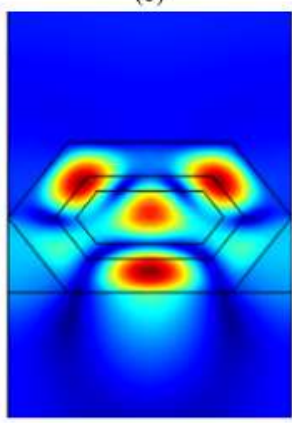

(d)
Fig. 6. TE electric field intensity profiles of the nonuniform NW at (a) 919 and (b) $673 \mathrm{~nm}$ and TM magnetic field intensity profiles of the non-uniform NW at (c) 716 and (d) $573 \mathrm{~nm}$.

The new resonances for TE polarizations are shown as the whispering-gallery modes in Figs. 6 (a) and (b) for the wavelengths of $919 \mathrm{~nm}$ and $673 \mathrm{~nm}$, respectively. Additionally, TM polarizations' cavity modes are seen in Figs. 6 (c) and (d) for the wavelengths of 716 and 573 $\mathrm{nm}$.

The $J_{S C}$ of the optimized non-uniform hexagonal NWSC is calculated as 9.28 $\mathrm{mA} / \mathrm{cm}^{2}$, which shows $14.3 \%$ enhancement compared to the uniform structure and a $59.60 \%$ increase compared to the thin film SC.

Since it is supposed to enhance the $J_{s c}$ by optimizing all layer dimensions, the PSO algorithm is used to optimize the a-Si layer position $(d), W_{1-3}$, and $U_{1-3}$. In this way, the $J_{s c}$ is computed as $9.401 \mathrm{~mA} / \mathrm{cm}^{2}$ that only shows a $1.32 \%$ increase compared to the non-uniform structure. We have named this structure the modified NWSC, and the optimized parameters of this structure alongside the nun-uniform structure are displayed in Table 1.

\section{IV.MulTiPle NWSCS}

In this section, multiple NWs with different cross-sectional geometries have been proposed to increase further the absorption spectra, shown in Fig. 7.

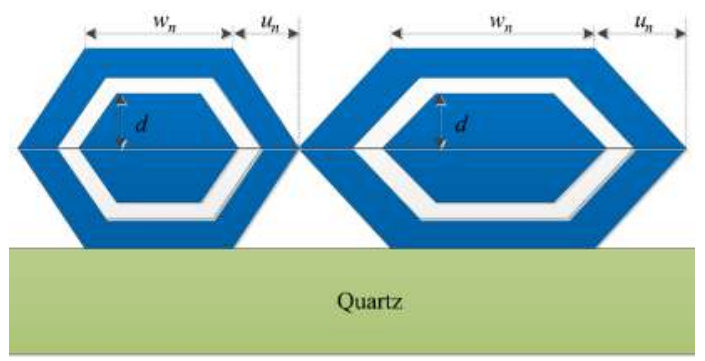

Fig. 7. Cross-sectional view of multiple structure composed of two non-uniforms NWs with different geometries.
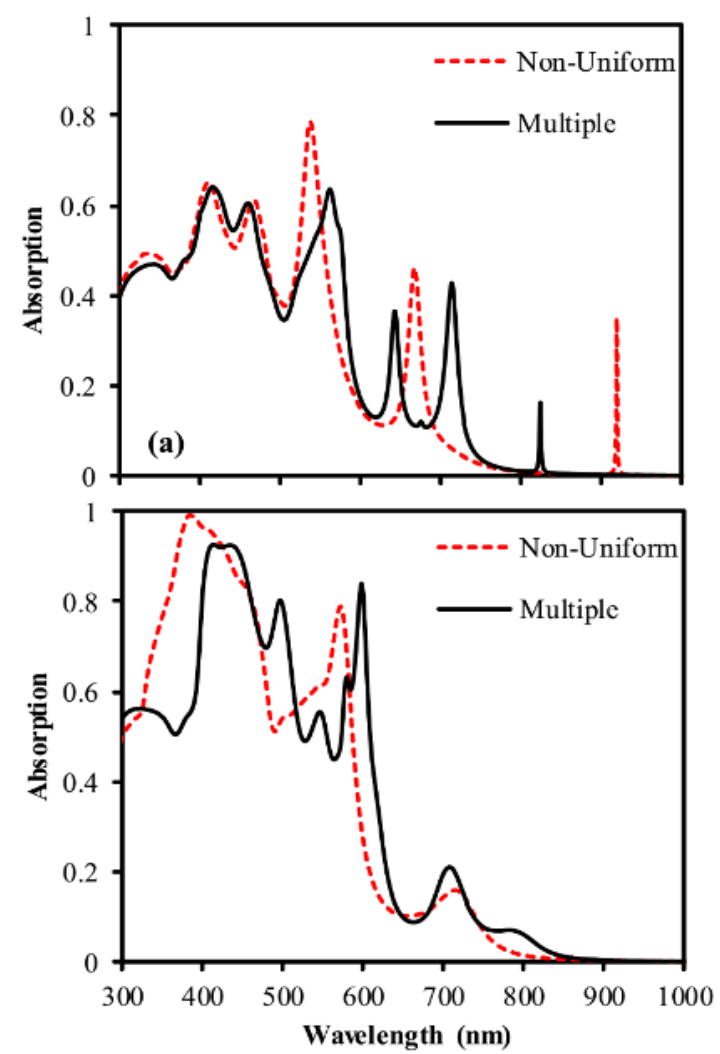

Fig. 8 Absorption spectra of non-uniform and multiple nanowire solar cells for (a) TE and (b) TM polarization. 
The proposed multiple NW structure is composed of two non-uniforms NWs with different cross-sectional geometries. An array of the above-mentioned multiple NWs is placed on the quartz substrate to simulate the SC.

Once more, to extract the maximum $J_{S c}$, the PSO algorithm is used to optimize the structure's geometry and dimensions. All the parameters can be found in Table 1 .

In both TE and TM polarizations, the absorption spectrum is obtained for the optimized multiple NWSC, and then it is compared to the absorption spectrum of the non-uniform NW structure in Fig. 8. The multiple structures offer more peaks in the absorption spectra, which results from resonances of different NWs.

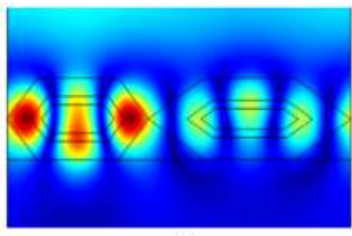

(a)

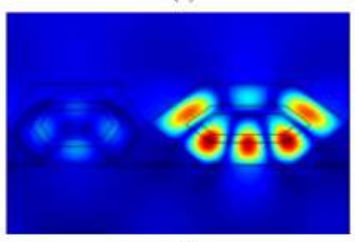

(c)

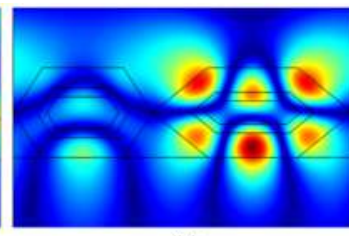

(b)

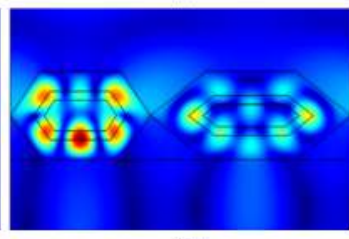

(d)
Fig. 9 TE electric field intensity profiles of the multiple NWs at (a) 824 and (b) $712 \mathrm{~nm}$ and TM magnetic field intensity profiles of the multiple NWs at (c) 597 and (d) $543 \mathrm{~nm}$.

The intensity and position of these resonances are changed compared to the non-uniform structure, as well. The $J_{s c}$ enhancement of this structure compared to the non-uniform and uniform structures is $5.8 \%$ and $17.99 \%$, respectively.

Fig. 9 shows the whispering-gallery modes in TE polarization at $824 \mathrm{~nm}$ and $712 \mathrm{~nm}$ wavelength. Additionally, the cavity modes are seen in 597 and $543 \mathrm{~nm}$ wavelengths for TM polarizations.

Once again, to determine the type of the resonance modes, the electromagnetic field intensity profile is shown in figure 9 , along with different absorption resonance positions in both $\mathrm{TE}$ and TM polarizations. In Table 1, the exact values of optimized parameters are listed for different proposed structures, along with the increase in $J_{s c}$ compared to the thin-film SC. Using multiple structure leads to enhancing the $J_{S C}$ of the SC by more than $68 \%$, which allows the implementation of high-efficiency ultrathin SCs.

Table 1. Optimal dimensions, Current densities, and related enhancements of different structures.

\begin{tabular}{|c|c|c|c|c|}
\hline \multirow{2}{*}{ Structure Uniform } & \multirow{2}{*}{$\begin{array}{c}\text { Nom- } \\
\text { Uniform }\end{array}$} & \multirow{2}{*}{ Modified } & \multicolumn{2}{|c|}{ Multiple } \\
\hline & & & NW (1) & NW (2) \\
\hline$d(\mathrm{~nm}) \quad 35$ & 55.08 & 46.80 & 54.34 & 45.37 \\
\hline$w_{1}(\mathrm{~nm}) 141.42$ & 144.12 & 158.88 & 120.2 & 158.72 \\
\hline$w_{2}(\mathrm{~nm}) 77.78$ & 123.20 & 170.34 & 141.22 & 172.4 \\
\hline$w_{3}(\mathrm{~nm}) 49.50$ & 95.52 & 89.32 & 94.28 & 104.87 \\
\hline$u_{1}(\mathrm{~nm}) 70.71$ & 72.06 & 42.91 & 24.07 & 32.78 \\
\hline$u_{2}(\mathrm{~nm}) 38.89$ & 61.60 & 74.92 & 37.86 & 58.62 \\
\hline$u_{3}(\mathrm{~nm}) 24.75$ & 47.76 & 75.77 & 68.91 & 129.21 \\
\hline $\begin{array}{l}J_{S C} \\
\left(\mathrm{~mA} / \mathrm{cm}^{2}\right)\end{array}$ & 9.278 & 9.401 & & \\
\hline $\begin{array}{l}\text { Enhance } 40.59 \\
(\%)\end{array}$ & 59.60 & 61.72 & & \\
\hline
\end{tabular}

\section{Conclusion}

The light absorption properties of the uniform, non-uniform, and multiple three-layer NWSCs are studied. Results have shown that the proper design of a non-uniform NW structure increases the absorption inside the solar for the wide range of wavelengths and generates new resonance modes. These effects can increase the $J_{S C}$ of the SCs, effectively. In order to increase the $J_{S c}$, dimensions of both nonuniform and multiple NWSCs were optimized. Using the optimal non-uniform and multiple NW structures resulted in a $14.3 \%$ and $17.99 \%$ increase in the $J_{S C}$ compared to the uniform structure with the same thickness.

\section{REFERENCES}

[1] K.B. Crozier, K. Seo, H. Park, A. Solanki, and S.-Q. Li, "Controlling the Light Absorption in a Photodetector Via Nanowire Waveguide Resonances for Multispectral and Color Imaging," IEEE J. Sel. Top. Quantum Electron. Vol. 24, pp. 1-12, 2018.

[2] S. Skalsky, Y. Zhang, JA. Alanis, HA. Fonseka, A.M. Sanchez, H. Liu, and P. Parkinson, "Heterostructure and Q-factor engineering for 
low-threshold and persistent nanowire lasing," Light Sci. Appl. Vol. 9, pp. 43-54, 2020.

[3] X. Yuan, X. Chen, X. Yan, W. Wei, Y. Zhang, and $\mathrm{X}$. Zhang, "Absorption-Enhanced UltraThin Solar Cells Based on Horizontally Aligned $\mathrm{p}-\mathrm{i}-\mathrm{n} \quad$ Nanowire Arrays," Nanomaterials, Vol. 10, pp. 1111 (1-10), 2020.

[4] G. Brönstrup, N. Jahr, C. Leiterer, A. Csáki, W. Fritzsche, and S. Christiansen, "Optical Properties of Individual Silicon Nanowires for Photonic Devices," ACS Nano, Vol. 4, pp. 7113-7122, 2010.

[5] G. Brönstrup, C. Leiterer, N. Jahr, C. Gutsche, A. Lysov, I. Regolin, W. Prost, F.J. Tegude, W. Fritzsche, and S. Christiansen, "A precise optical determination of nanoscale diameters of semiconductor nanowires," Nanotechnol. Vol. 22, pp. 385201 (1-10), 2011.

[6] P. Spinelli, M.A. Verschuuren, and A. Polman, "Broadband omnidirectional antireflection coating based on subwavelength surface Mie resonators," Nat. Commun. Vol. 3, pp. 692 (15), 2012.

[7] L. Wen, Z. Zhao, X. Li, Y. Shen, H. Guo, and Y. Wang, "Theoretical analysis and modeling of light trapping in high efficicency GaAs nanowire array SCs," Appl. Phys. Lett. Vol. 99, pp. $143116(1-4), 2011$.

[8] L. Cao, J.S. White, J.-S. Park, J.A. Schuller, B. M. Clemens, and M.L. Brongersma, "Engineering light absorption in semiconductor nanowire devices," Nat. Mater. Vol. 8, pp. 643647, 2009.

[9] K. Seo, M. Wober, P. Steinvurzel, E. Schonbrun, Y. Dan, T. Ellenbogen, and K.B. Crozier, "Multicolored Vertical Silicon Nanowires," Nano Lett. Vol. 11, pp. 18511856, 2011.

[10]H. Park and K.B. Crozier, "Elliptical silicon nanowire photodetectors for polarizationresolved imaging," Opt. Express, Vol. 23, pp. 7209-7216, 2015.

[11]A.R. Jha, Solar Cell Technology and Applications, CRC Press, 2009.

[12]D. Muñoz, T. Desrues, and P.-J. Ribeyron, $a$ Si:H/c-Si Heterojunction Solar Cells: A Smart Choice for High Efficiency Solar Cells, in Physics and Technology of AmorphousCrystalline Heterostructure Silicon Solar Cells, W.G.J.H.M. van Sark, L. Korte, F. Roca, Eds. Berlin, Heidelberg: Springer, 2012.
[13]P. Krogstrup, H.I. Jørgensen, M. Heiss, O. Demichel, J.V. Holm, M. Aagesen, J. Nygard, and A.F. i Morral, "Single-nanowire solar cells beyond the Shockley-Queisser limit," Nat. Photon. Vol. 7, pp. 306-310, 2013.

[14] S.-K. Kim, K.-D. Song, T.J. Kempa, R.W. Day, C.M. Lieber, and H.-G. Park, "Design of Nanowire Optical Cavities as Efficient Photon Absorbers," ACS Nano, Vol. 8, pp. 3707-3714, 2014.

[15] Y. Xing, K. Zhang, J. Zhao, P. Han, Z. Yang, Y. Yuan, and Q. Ding, "Antireflection and absorption properties of silicon parabolicshaped nanocone arrays," Optik, Vol. 128, pp. 133-138, 2017.

[16] K.T. Fountaine, C.G. Kendall, and H.A. Atwater, "Near-unity broadband absorption designs for semiconducting nanowire arrays via localized radial mode excitation," Opt. Express, Vol. 22, pp. A930-A940, 2014.

[17] O.H.A. Zoubi, T.M. Said, M.A. Alher, S.E. Ghazaly, and H. Naseem, "Broadband high efficiency silicon nanowire arrays with radial diversity within diamond-like geometrical distribution for photovoltaic applications," Opt. Express, Vol. 23, pp. A767-A778, 2015.

[18] B.C. Sturmberg, K.B. Dossou, L.C. Botten, A.A. Asatryan, C.G. Poulton, R.C. McPhedran, C. Martijn de Sterke, "Nanowire array photovoltaics: Radial disorder versus design for optimal efficiency," Appl. Phys. Lett. Vol. 101, pp. 173902 (1-4), 2012.

[19]B. Wang, E. Stevens, and P.W. Leu, "Strong broadband absorption in GaAs nanocone and nanowire arrays for solar cells," Opt. Express, Vol. 22, pp. A386-A395, 2014.

[20] M. Hussein, M.F.O. Hameed, N.F. Areed, and S.S.A. Obayya, "Ultra-high efficient solar cell based on decagonal arrays of silicon nanowires," Opt. Eng. Vol. 53, pp. 117105 (17), 2014.

[21] B. Wang and P.W. Leu, "Enhanced absorption in silicon nanocone arrays for photovoltaics," Nanotechnol. Vol. 23, pp. 194003 (1-7), 2012.

[22] J. Tang, Z. Huo, S. Brittman, H. Gao, and P. Yang, "Solution-processed core-shell nanowires for efficient photovoltaic cells," Nat. Nanotechnol. Vol. 6, pp. 568-572, 2011.

[23] B. Tian, X. Zheng, T.J. Kempa, Y. Fang, N. Yu, G. Yu, J. Huang, and C.M. Lieber, "Coaxial silicon nanowires as solar cells and 
nanoelectronic power sources," Nature Vol. 449, pp. 885-889, 2007.

[24]S.-K. Kim, R.W. Day, J.F. Cahoon, T.J. Kempa, K.-D. Song, H.-G. Park, and C.M. Lieber, "Tuning light absorption in core/shell silicon nanowire photovoltaic devices through morphological design," Nano Lett. Vol. 12, pp. 4971-4976, 2012.

[25] K.-D. Song, T.J. Kempa, H.-G. Park, and S.-K. Kim, "Laterally assembled nanowires for ultrathin broadband solar absorbers," Opt. Express, Vol. 22, pp. A992-A1000, 2014.

[26] M. Adachi, M. Anantram, and K. Karim, "Optical properties of crystalline- amorphous core- shell silicon nanowires," Nano Lett. Vol. 10, pp. 4093-4098, 2010.

[27]L.-F. Cui, R. Ruffo, C.K. Chan, H. Peng, and Y. Cui, "Crystalline-amorphous core- shell silicon nanowires for high capacity and high current battery electrodes," Nano Lett. Vol. 9, pp. 491-495, 2008.

[28]Z. Yang, X. Li, D.Y. Lei, A. Shang, and S. Wu, "Omnidirectional absorption enhancement of symmetry-broken crescent-deformed singlenanowire photovoltaic cells," Nano Energy, Vol. 13, pp. 9-17, 2015.

[29]T. Khudiyev and M. Bayindir, "Nanosprings harvest light more efficiently," Appl. Opt. Vol. 54, pp. 8018-8023, 2015.

[30]M. Shahraki, M.R. Salehi, and E. Abiri, "Optimal design of laterally assembled hexagonal silicon nanowires for broadband absorption enhancement in ultrathin solar cells,” Opt. Eng. Vol. 54, pp. 115102-115102, 2015.

[31]N.F. Areed, M. Fouad, and S.S. Obayya, "Highly Efficient Solid Gear-Shaped Silicon Nanowire for Solar Energy Harvesting," IEEE Photonics Technol. Lett. Vol. 29, pp. 205-208, 2016.

[32] M. Hussein, M.F.O. Hameed, N.F. Areed, A. Yahia, and S. Obayya, "Funnel-shaped silicon nanowire for highly efficient light trapping," Opt. Lett. Vol. 41, pp. 1010-1013, 2016.

[33] J. Yao, H. Yan, and C.M. Lieber, "A nanoscale combing technique for the large-scale assembly of highly aligned nanowires," Nat. Nanotechnol. Vol. 8, pp. 329-335, 2013.

[34]L. Cao, P. Fan, A.P. Vasudev, J.S. White, Z. Yu, W. Cai, J.A. Schuller, S. Fan, and M.L.
Brongersma, "Semiconductor nanowire optical antenna solar absorbers," Nano Lett. Vol. 10, pp. 439-445, 2010.

[35] M. Shahraki, M.R. Salehi, and E. Abiri, "Omnidirectional broadband absorption enhancement in laterally assembled quadrangle silicon nanowire solar cells," J. Mod. Opt. Vol. 64, pp. 23-31, 2017.

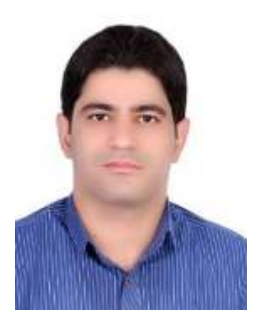

Mojtaba Shahraki received the B.Sc. degree in electronics engineering from the University of Sistan and Baluchestan (USB), Zahedan, Iran, and the M.Sc. and $\mathrm{PhD}$. degrees in electronics engineering from Shiraz University of Technology (SUTECH), Shiraz, Iran, in 2009, 2012 and 2017, respectively. He is now working with University of Sistan and Baluchestan and His current research explores design, simulation, modeling, and optimization of light trapping structures in ultrathin solar cells.

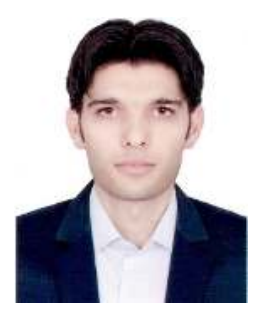

Majid Ghadrdan He received the B.Sc., M.Sc. and $\mathrm{PhD}$. degrees in electronics engineering in electronics engineering from the University of Sistan and Baluchestan (USB), Zahedan, Iran, in 2010, 2012 and 2018, respectively.

He is currently assistant professor at University of Sistan and Baluchestan. His interests include Photonic crystals, Plasmonics, Nonlinear optics, and Nanophotonics. 